\title{
METHODS OF RADIO-FREQUENCY CURRENT DRIVE
}

\author{
N. J. FISCH* \\ Princeton Plasma Physics Laboratory, Princeton, New Jersey 08543
}

Received April 3, 2013

Accepted for Publication June 10, 2013

doi:10.13182/FST13-670

Radio-frequency waves can penetrate thermonuclear plasmas, depositing momentum and energy with great selectivity: in select resonant ions or electrons, in select resonant regions, and with select momentum. When these waves are injected asymmetrically with respect to the toroidal direction in tokamaks, they can drive a toroidal electric current. The advantage of driving this current by waves is that a tokamak reactor might then be operated in the steady state. This lecture will review the elementary processes of wave-particle interactions in plasma that underlie the current drive effect.

KEYWORDS: noninductive current drive, $r f$ waves, $r f$ current drive

Note: Some figures in this lecture may be in color only in the electronic version.

\section{INTRODUCTION}

Using radio-frequency (rf) waves to drive the toroidal current in tokamak reactors is attractive as a means to achieve steady-state tokamak operation. The toroidal current is necessary for tokamak confinement. The original conception of the tokamak anticipated that the toroidal current would be driven by a direct-current (dc) toroidal electric field. The dc electric field would be induced by a time-varying magnetic field. Indeed, since the dc electric field, being toroidal, necessarily has curl, it implies the presence of a time-varying magnetic field. To drive a current of one toroidal sign then requires a monotonically time-varying magnetic field. Since only finite magnetic fields can be produced, this process cannot persist in the steady state. At some point, the magnetic field must cease to increase, the dc electric field then vanishes, and with that the poloidal magnetic field vanishes as well, and charged particles are no longer confined.

However, rf wave power, when injected asymmetrically with respect to the toroidal direction in a tokamak, can drive a steady-state toroidal electric current (see Fig. 1). One way to drive the current is by interacting asymmetrically

*E-mail: fisch@princeton.edu with resonant electrons. The toroidal current then supports the poloidal magnetic field. One can imagine that the waves just push electrons in the toroidal direction against some sort of friction with ions; as long as the power remains on, the current persists. Thus, even though the current is not curl-free, it may persist in the steady state when not driven by a dc electric field.

The capability to operate the tokamak in the steady state with acceptable circulating power is thought to be important, if not critical, for economical fusion power through the tokamak approach. The power cost remains large in contemporary designs but might still allow economical fusion power. In contemporary designs, the rf-driven current does not provide the full toroidal current, since much of the current is already provided by the socalled bootstrap current. Yet, it remains important both to find the highest efficiency for current drive by waves and to design tokamaks that best utilize the rf-driven currents. In addition, other uses have been found for the steadystate currents produced by rf waves, such as to control heat and particle transport or to stabilize magnetohydrodynamic instabilities.

There have been a variety of mechanisms proposed to drive noninductive current, both by neutral beams and by waves. Neutral beams drive current when they become 


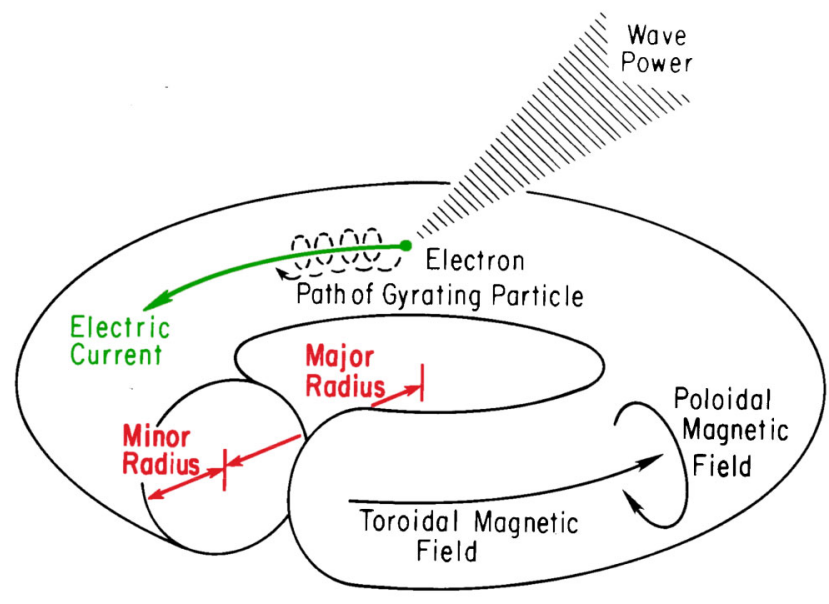

Fig. 1. Radio-frequency waves injected asymmetrically into a tokamak.

ionized in a plasma, but the efficiency of neutral-beamdriven current tends to be less than that of current driven by the most efficient of rf means. It is also the case that the technology for producing if waves tends to be more compatible with a reactor environment than the technology for producing neutral beams. Nonetheless, neutral beams remain a viable method at least for providing part of the toroidal current in a tokamak reactor.

Magnetized plasma supports many different kinds of plasma waves-and different waves feature different kinds of wave-particle interactions. The efficiency of current production by different waves is also different. This lecture will explain at an elementary level the wave methods of producing this current. Further details, the historical context, and the early experiments demonstrating these methods can be found in a review paper by the present author. ${ }^{1}$ Recent reviews of noninductive current drive methods for educational purposes can also be found. ${ }^{2,3}$ However, we shall also cite many of the original papers where these methods were first proposed.

\section{LOWER HYBRID CURRENT DRIVE}

The most successful technique to date is to drive these currents by lower hybrid waves. ${ }^{4}$ In lower hybrid current drive (LHCD), the current is carried by a tail of superthermal electrons. A superthermal electron interacts with an electrostatic wave such that it is pushed in the toroidal direction. The way this happens is through Landau damping of the electrostatic wave. Further exploration of the wave-particle interaction can be found, for example, in the classic book by Stix, ${ }^{5}$ the more recent book by Brambilla, ${ }^{6}$ the most recent very excellent book by $\operatorname{Rax}^{7}$ or (put more briefly) in the lecture in this issue by $\operatorname{Rax}^{8}$

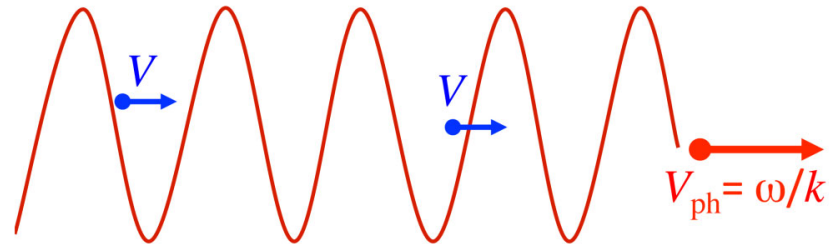

Fig. 2. Resonant interaction of electrons with an electrostatic wave. The solid wavy line indicates the wave potential as a function of distance along the direction of the phase velocity at a given instant of time.

Consider the interaction of an electrostatic wave with frequency $\omega$ and wave number $k$ traveling to the right as in Fig. 2. For this purpose, the wave could be a Langmuir wave; it will operate similarly to the lower hybrid wave, which is nearly electrostatic. Electrons resonant with the wave, i.e., those moving near the wave phase velocity, are pushed, while the nonresonant electrons are not pushed. In order for this to happen, the wave must be resonant, that is, $\omega-\mathbf{k} \cdot \mathbf{v}=0$. The push can be either to increase the particle velocity or to decrease it, depending on the phase of the particle in the trough of the wave. To the extent that both processes occur, the effects will simply cancel.

However, for distribution functions near collisional equilibrium, namely, nearly Maxwellian in energy, there will be more electrons that get pushed to higher energy than those that get pushed to lower energy. This is the principle of Landau damping of the wave. It is also the principle of the current drive effect, since we can now just focus on those excess slower electrons that are pushed to higher velocities. Note also that in this picture it is not possible to push particles to slower velocities, on average, since, under diffusion, the push is always toward the lower populated states, which are at higher energy.

So, consider an electron moving with velocity $v$ in the direction of the wave phase velocity that gets pushed to $v+\Delta v$. The extra instantaneous current density carried by a density $n$ (each with charge $q$ ) of these electrons resonant with the wave may be written as

$$
J=q n \Delta v .
$$

The energy density required to accomplish this current for small $\Delta v$ is

$$
E=n m v \Delta v .
$$

At first glance, it may seem that to get high current with less energy, it would be advantageous to employ waves that push the slower electrons. After all, the momentum or current gained is only proportional to $\Delta v$, whereas the energy expended is proportional to $v \Delta v$. However, what is important is not the instantaneous current that would be produced, but how long this current 
would persist. This current would have to be renewed every collision time of the resonant particles or with frequency $v(v)$. It is important to note the velocity dependence of this collision frequency, because it is very sensitive to velocity. Thus, the power density dissipated $P_{D}$ is the energy expended per unit time to maintain the current $J$, or

$$
P_{D}=v(v) E,
$$

and the current drive efficiency may be put as

$$
J / P_{D}=q / m v v(v) .
$$

Now for superthermal electrons, the collision frequency goes as $v^{-3}$. This is a crucial feature of Coulomb collisions, for it enables the high efficiency of current drive by superthermal electrons, which may have velocities about four or five times the thermal velocity. This collisional effect would then reduce the power dissipated by about a factor of $10^{2}$ over what might have been thought based on the energy argument. Thus, by Eq. (4), the current drive efficiency using lower hybrid waves goes as $v^{2}$. This current drive effect has been routinely demonstrated in detail on many tokamaks, with up to mega-amps of current being produced with megawatts of lower hybrid wave power.

Another notable feature of the current drive efficiency that can be seen through Eq. (4) is the dependence of the efficiency on plasma density. Since the collision frequency $\nu$ is proportional to density, the efficiency is inversely proportional to density. This feature of the current drive effect has also been demonstrated routinely using lower hybrid waves on many tokamaks. We may contrast this feature of $\mathrm{rf}$ current drive with the conductivity seen by currents driven by a dc electric field. The dc conductivity of a plasma is independent of density, since, on the one hand, larger density implies more scattering centers, but, on the other hand, because all electrons are pushed equally regardless of velocity, and more electrons share the burden of carrying the current, the electrons get pushed at lower average (drift) velocities. For rf-driven currents, however, the speed of the electrons receiving a push is fixed, usually by a resonance condition.

Let us go over some of the assumptions made in deriving Eq. (4). To determine the efficiency of this effect, we assumed that we knew which electrons were pushed. But, as the wave interacts with the distribution of electrons, this distribution function changes in response to the wave excitation. How do we then know with which electrons the wave interacts? It turns out that, even though the distribution function does become distorted, the velocity of the resonant electrons can be precisely inferred.

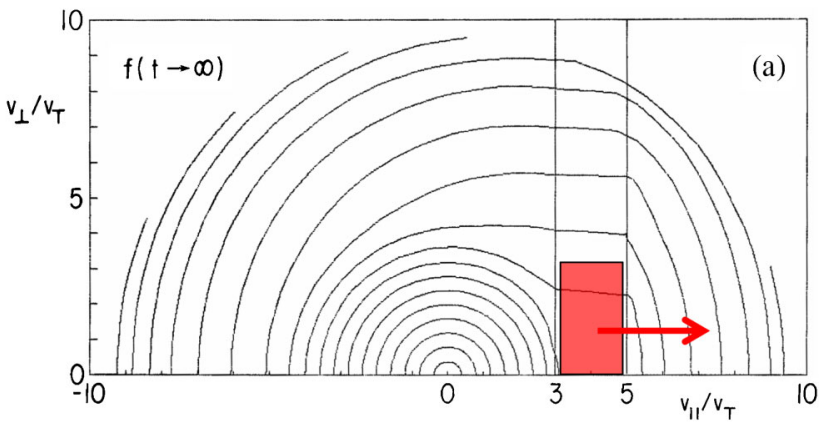

Fig. 3. Contour plot of the electron distribution in $v_{\|}-v_{\perp}$ space under lower hybrid wave excitation. The shaded region shows where most of the wave-particle interactions take place, with the arrow indicating the direction of the net wave push on resonant electrons. (Adapted from Ref. 9.)

In Fig. 3, we show a contour plot of the electron distribution function in $v_{\|}-v_{\perp}$ space, under the condition that intense resonant waves were launched, but only with parallel phase velocities between three and five times the thermal velocity $v_{T}$. The velocity $v_{\|}$is parallel to the direction of the wave phase velocity, and $v_{\perp}$ is perpendicular to this direction. The current is to be generated in the direction of the wave phase velocity. In a tokamak, the toroidal magnetic field would be in the direction of $v_{\|}$. This contour plot was produced by solving the Fokker-Planck equation, which models the collisional effects, together with a diffusion equation, which models the rf interaction. ${ }^{9}$

What can be seen from this contour plot is that the distribution function is Maxwellian for thermal velocities but is highly distorted from a Maxwellian for superthermal velocities, mainly in the parallel direction but also in the perpendicular direction. Clearly, there is a current drive effect, since the distortions in the parallel direction from a Maxwellian are highly asymmetric.

However, we can also tell from Fig. 3 with which electrons the wave interacts. The wave only interacts with electrons in the resonant velocity region, namely, those in the range $3<v_{\|} / v_{T}<5$. Hence, the parallel velocity of the resonant electrons is known to the extent that the wave spectrum is limited in parallel phase velocity. And, within that region, most of the electrons have perpendicular velocities smaller than the parallel velocities, so that most of the wave-particle interactions take place in the shaded region in Fig. 3. Were the wave resonant region narrower in parallel velocity space, then not only would this shaded region too be narrower in parallel velocity space but also its extent in the perpendicular direction would be less.

Thus, since the perpendicular velocity is negligible compared with the parallel velocity of most electrons being pushed, for a reasonably narrow region of velocity space, we know very well the velocity of the electrons that 
are being pushed. That velocity is mainly their parallel velocity, which is the wave phase velocity. Hence, we can use Eq. (4) with some confidence that we know the velocity $\mathbf{v}$.

There is another assumption implicit in the use of Eq. (4), and that is that the time it takes an electron to lose its directed velocity is dependent only on its initial velocity coordinate and not on the wave intensity. The justification for this assumption is that, during most of the time over which an electron slows down, it is nonresonant with the wave.

A related assumption implicit in the use of Eq. (4) is that the electrons that are pushed remain in the plasma and carry current as long as they are not slowed down by collisions. This assumption would be violated for the socalled trapped electrons in tokamaks that do not carry toroidal current. This assumption would also be violated if somehow, through effects not considered here, the electrons that were pushed were lost before they slowed down by collisions.

Yet another assumption implicit in the use of Eq. (4) is that we in fact know how to calculate $\nu(v)$. We could use the one-dimensional (in velocity space) slowing-down formula for electrons (as was originally done in Ref. 4), but it is not so hard to get this answer exactly, at least in the high-velocity limit. That can be done by considering both slowing down in the parallel direction as well as pitch angle scattering in the perpendicular direction. When we do that, we find that efficient current drive is also possible using electron cyclotron waves, ${ }^{10}$ wherein electrons are pushed by the wave only in the perpendicular direction, as we will describe in Sec. III.

From Eq. (4), it can also be seen that there is another regime of relatively high efficiency, namely, where electrons would be pushed with low (subthermal) parallel velocities but at least thermal perpendicular velocities. ${ }^{11,12}$ If the perpendicular velocities were around the thermal velocity, and if only low parallel velocity electrons were pushed, then the collision frequency would be roughly independent of the parallel velocity, while the energy expended to produce a velocity increment would be proportional to the parallel velocity. Thus, in this regime, the efficiency would go as $1 / v_{\|}$. A wave with low parallel phase velocity could be an Alfvén wave. How these waves might be used in practice to generate substantial current in a tokamak remains an issue, however.

\section{ELECTRON CYCLOTRON CURRENT DRIVE}

Another method that has high efficiency is electron cyclotron current drive (ECCD). The mechanism for current drive can be seen from Fig. 4, which depicts what happens when an electron is pushed in the perpendicular velocity direction but not in the parallel velocity direction. The electron absorbs no parallel momentum, but in going

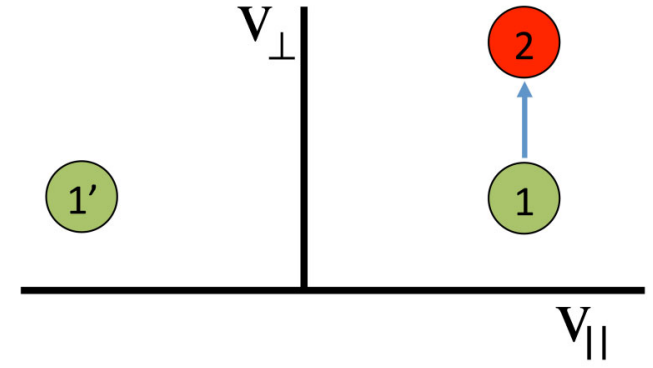

Fig. 4. An electron is pushed by a wave from velocity space location 1 to velocity space location 2 , with no input of parallel momentum. Note that the symmetry is broken, since the symmetrically counter-propagating electron at velocity location $1^{\prime}$ is not pushed.

from velocity space location 1 to velocity space location 2 , because it becomes more energetic, it collides less both with ions and with the slower electrons. This creates an asymmetry. If half of the electrons are going to the left and half are going to the right (in the parallel direction), but only those that are going to the right gain perpendicular energy from a wave spectrum, then those going to the right will persist in going to the right, while those going to the left will slow down more quickly on ions (on electrons too, but those collisions, for nonrelativistic electrons, are current-conserving). Hence, a net flow of electrons persists in going to the right. Note that the ions collide more with the electrons going to the left. Hence, the ions tend to go to the left, so that the electron flow going to the right is balanced by the ion flow going to the left. In such a manner, even with no parallel momentum input by the waves, current is nonetheless generated, while particle momentum is conserved.

One can plot the expected value of the current carried by an electron that begins in velocity space location 1 compared with that in velocity space location 2 (see Fig. 5). It is an expected value, since collisions give random kicks. But, on average, an electron beginning in velocity space location 2 carries its directed momentum longer than an electron beginning in velocity space location 1. Note that, as the electron slows down, it loses its expected directed velocity more rapidly, since it collides more often as it becomes slower.

The difference between these two curves is the current drive effect of the push in the perpendicular direction. Thus, initially, there is no net current, since the electrons start out with the same parallel velocity. Also, at very large times, the directed velocities of the electrons vanish, so there is no net current. But, the current drive effect becomes large over intermediate times, namely, on the order of a collision time.

Thus, Fig. 5 suggests that we can assign to every point in $v_{\|}-v_{\perp}$ space a certain potential for carrying current over all of time. We can consider a wave that pushes particles in an arbitrary direction in velocity space, 


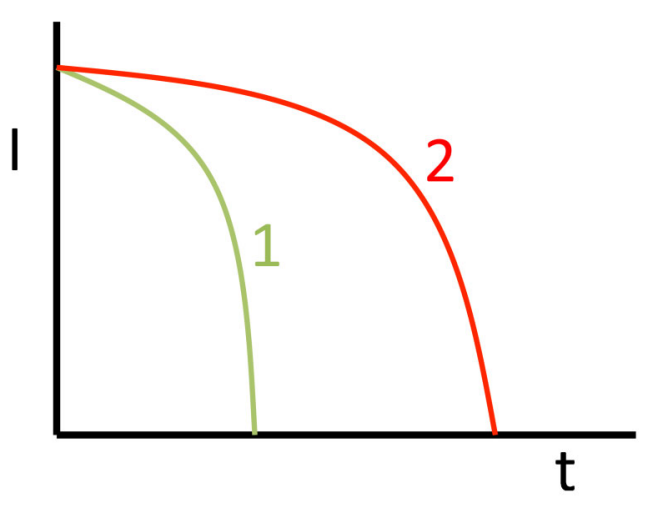

Fig. 5. Expected current $I$ of an electron that starts out in velocity space location 1 or 2 as a function of time. Current decreases in time owing to collisions.

neither purely in the parallel direction (like for lower hybrid waves) nor purely in the perpendicular direction. Then, by moving a particle incrementally from one location to another in the direction $\mathbf{S}$ (see Fig. 6), we change this potential, at the expense of the energy expended. We call this potential for the current the Green's function for the current drive. In the steady state, we can thus write the current drive efficiency in the form ${ }^{10}$

$$
\frac{J}{P_{d}}=\frac{\mathbf{S} \cdot(\partial / \partial \mathbf{v}) \chi(\mathbf{v})}{\mathbf{S} \cdot(\partial / \partial \mathbf{v}) \epsilon(\mathbf{v})},
$$

where $\mathbf{S}$ is the unit vector in the direction of the rfinduced flux, $\chi(\mathbf{v})$ is the Green's function for the current drive, $\mathbf{v}$ is the velocity of the resonant electrons, i.e., the vicinity in velocity space of the induced flux, and $\epsilon(\mathbf{v})=m v^{2} / 2$ is the kinetic energy of the resonant electrons. The function $\chi(\mathbf{v})$ takes a particularly simple form in the high-velocity limit $\left(v \gg v_{T}\right)$ :

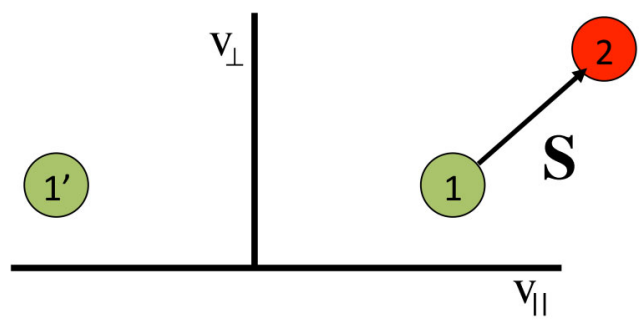

Fig. 6. An electron is pushed by a wave from velocity space location 1 to velocity space location 2 , along velocity space direction $\mathbf{S}$. Note that the symmetry is broken, since the symmetrically counter-propagating electron at velocity location $1^{\prime}$ is not pushed.

$$
\chi(\mathbf{v})=\frac{e v^{3} v_{\|}}{\Gamma\left(5+Z_{i}\right)},
$$

where $Z_{i}$ is the ion charge state and $\Gamma=n e^{4} \ln \Lambda / 4 \pi \epsilon_{0} m^{2}$ is a measure of the collision frequency. (For the relativistic limit, see Refs. 13 through 15.)

Interestingly, it can be seen from Eqs. (5) and (6) that the ion charge state $Z_{i}$ only enters in the form $\left(5+Z_{i}\right)$. Only when it exceeds about 5 does it become important. The insensitivity to the charge state arises because the fast electrons, while they lose momentum to both ions and electrons, lose energy only to thermal electrons, which then allows them to lose momentum faster. Indeed, this is related to a second important message of Eqs. (5) and (6), which concerns the efficiency of pushing electrons already moving at superthermal speeds in the parallel direction, such as for LHCD. The message is that the efficiency depends little on the direction of $\mathbf{S}$. Although it was originally thought that parallel momentum input was necessary for LHCD, energy input is more important. In fact, it can be seen from Eqs. (5) and (6) that pushing these electrons in the perpendicular direction rather than in the parallel direction gives exactly three-quarters the efficiency.

One refers to Eq. (5) as an equation for the current drive efficiency. This very powerful equation for the efficiency relies upon knowing the velocity phase-space position of resonant electrons. Each point in the velocity space of the electrons was assigned a utility function in terms of the ability of an electron, initialized at that velocity, to retain its current.

This suggests that there may be other properties that might be similarly usefully attributed to phase-space position too. For example, instead of talking about a runaway electron (for definition of runaway, see Sec. IV), one can assign a runaway probability function to each point in velocity space. ${ }^{16}$ Then, by considering the rfdriven flux of electrons in velocity space, say, from velocity space location 1 to velocity space location 2 , we can calculate the incremental production of runaways. The runaway production rate per unit rf power would then be a function of velocity space just as the current drive efficiency is.

\section{EXPERIMENTAL VERIFICATION}

The intensive experimental investigation of the LHCD effect demonstrated not only the effect itself but also the underlying theoretical assumptions. In particular, the very close agreement between theory and experiment showed that the superthermal electrons could be described by classical collision theory and that they were not affected significantly by some anomalous collective effect.

Before these experiments were done, there was a question as to whether classical effects would dominate. It 
was not sufficient that parallel Spitzer resistivity had been demonstrated, because that resistivity is an integrated effect over all electrons and is hence dominated by the effects of the thermal electrons. On the other hand, rf current drive depends sensitively on the dynamics of the relatively small percentage of the electron population resonant with the wave. At the same time, since the theory depends sensitively on the wave phase velocity, much more information about the underlying physics could be inferred from determining the efficiency as a function of the wave phase velocity compared with the confirmation of an integrated theory, like the Spitzer conductivity.

Interestingly, the confirmation in detail of the current drive theory was actually made easier by some puzzling experimental results. In these experiments, the so-called ramp-up experiments, the toroidal current was increased as a function of time, or ramped up due to the rf waves. The increase in current led to an increase in the poloidal magnetic field energy. In fact, as much as $40 \%$ of the rf power was converted into poloidal field energy. ${ }^{17}$ At first, that seemed like an astoundingly high fraction.

In fact, the same theoretical approach that predicted the current drive efficiency could be extended to account for a dc electric field. The toroidal dc electric field is induced by the ramp-up of the magnetic field, which, to a first approximation, can be taken to be increasing linearly in time. Thus, it was possible to calculate the rf-induced conductivity, namely, the term bilinear in the rf power and in the dc electric field strength. ${ }^{18}$ This explained how so much wave energy could end up in poloidal magnetic field energy. ${ }^{19}$ Moreover, the fact that the energy conversion could be so efficient, consistent with the theory, was strong evidence for the theory itself.

However, the most detailed data came from the full series of the PLT (Princeton Large Torus) series of current drive and ramp-up experiments. ${ }^{20}$ These experiments spanned several parameter regimes, leading to different physics regimes too, including that of steady-state current drive, ramp-up of the current, and even the unsuccessful sustainment of the current. In Fig. 7, we show the experimental data from PLT as plotted by Karney et al. ${ }^{20}$ In this plot, an attempt was made to check the theory of the electron dynamics without making many assumptions concerning the details of either the theory of wave propagation or wave damping. This was accomplished by comparing dimensionless quantities, each of which depended upon the power being absorbed.

Thus, the $x$-axis measures the ratio of the wave phase velocity $v_{p h}$ to the so-called runaway velocity $v_{R}$. The runaway velocity is the velocity that is so large that an electron can "run away" since Coulomb collisions cannot prevent it from being accelerated to yet larger velocities. Thus, the case of small electric fields, or nearly steadystate current drive, is described where $v_{p h} / v_{R}$ is small. The $y$-axis measures the efficiency of producing magnetic field energy or, in other words, the ratio of the power going

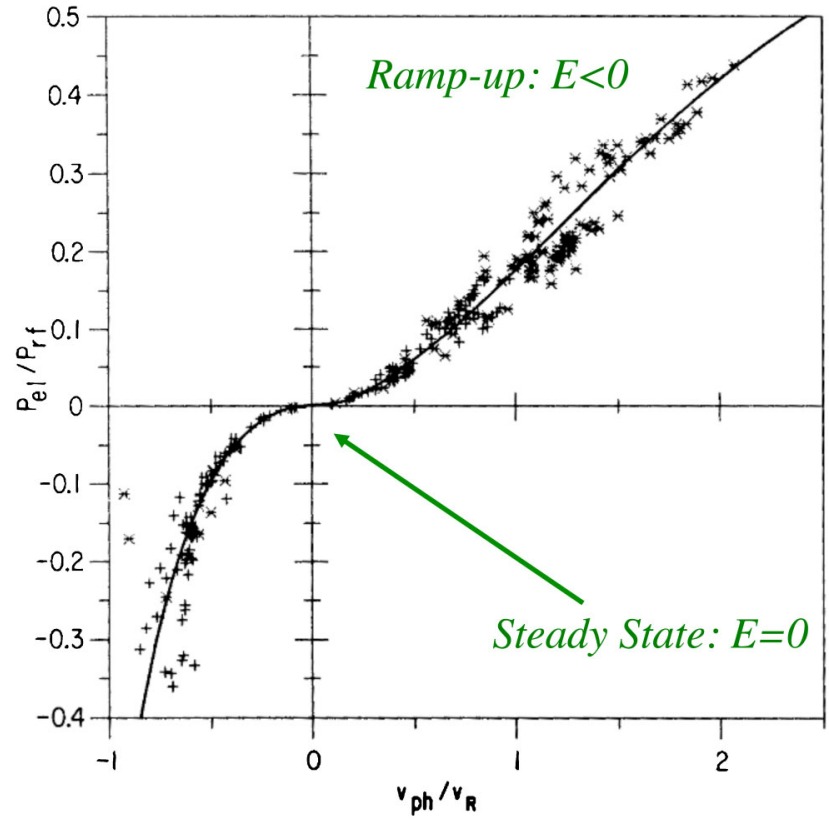

Fig. 7. Plot of $P_{e l} / P_{\mathrm{rf}}$ versus $v_{p h} / v_{R}$ for 273 PLT shots. The regime of steady-state current drive is described near the origin. The ramp-up regime is described in the upper right quadrant. (Adapted from Ref. 20.)

into the magnetic field to the rf power absorbed. When no power goes into the magnetic field, i.e., when there is no time-varying magnetic field, clearly the value of the power going into the magnetic field is zero, and so, the efficiency also vanishes. If the magnetic field is not timevarying, then the electric field vanishes. Hence, the origin in Fig. 7 describes steady-state current drive. The region near the origin describes the regime of rf-induced conductivity, sometimes called the hot conductivity regime.

The most interesting regime is the upper right quadrant in Fig. 7, which describes the current ramp-up regime. The faster the ramp-up, the larger the electric field that opposes the current rise, so $v_{p h} / v_{R}$ is large. On the other hand, the larger the electric field, the greater the efficiency of converting rf energy to magnetic field energy. This is because, when the electric field is small, current carriers slow down through collisions, which puts no energy into the magnetic field. But, when the electric field is large, the current carriers are slowed down by this field, which acts to oppose the rf-driven current, so that power flows from the particles to the fields. Plotted in terms of these dimensionless parameters, over 250 shots were tabulated. The only free parameters were the fraction of rf power absorbed and the upshift in parallel wave number that would enable this absorption in the plasma. (The upshift in parallel wave number remains a bit of a mystery even today, as there are multiple theories for explaining its existence. The upshift must take place in 
order that the wave energy be absorbed, since waves with too high a phase velocity would not interact resonantly with any electrons.)

With hardly any free adjustable parameters, the fit to the theoretical prediction was remarkable. (It is even remarkable that, when plotted in terms of these dimensionless parameters, all the data line up, rather than being scattered.) This experiment offered unmistakable proof not only of the current drive effect itself (both in the steady state and in the presence of a dc electric field) but also of the assumptions about Coulomb collisions upon which that theory was derived. The electron dynamics of fast electrons behaved as predicted classically, leaving little room for anomalous effects. This statement on the nature of the electron dynamics is farther reaching than any that could be offered through a detailed study of Spitzer resistivity, which is an integrated quantity. This important experiment was repeated on many other tokamaks, which confirmed the results.

The ECCD effect was also verified. For a fine review of the experimental verification, see Ref. 21. However, the description of the ECCD effect as a function of velocity space is more difficult, since it is harder to pinpoint the velocity space location of the resonant electrons. As an electron cyclotron wave traverses the plasma, it encounters different strength magnetic fields, making electrons of differing parallel velocities resonant. In contrast, for the lower hybrid wave, the parallel velocity resonance condition is largely determined by the waveguide phasing.

\section{MINORITY-SPECIES CURRENT DRIVE}

The method of generating current by electron cyclotron waves can be extended to ions as well, as long as there are ions of two differing ion charge states. Suppose first that by some magical means the ions of one of the charge states are maintained with a net toroidal velocity that differs from that of the ions of the other ion charge state. Consider now the frame of reference in which the ion current vanishes. In this frame of reference, the two species of ions are oppositely directed in velocity. Also, in this frame of reference, the electrons will try to follow the ions with higher ion charge state, since the electrons collide more frequently with those ions. As a result, the electrons acquire a drift in the direction of the ions with the higher ion charge state. In this frame of reference, then, there is a net current. Since the plasma is overall neutral, the current is frame-invariant, which means that there is a current in the laboratory frame as well, opposite in direction (since the electrons are negatively charged) to the current carried by the ions with higher ion charge state.

This is, in fact, the method of current drive by neutral beams, eluded to previously, which requires two species of ions. ${ }^{22}$ In neutral-beam current drive, one species of ions is tangentially injected as neutrals into the tokamak, where, upon ionization, the ion species produced has a different toroidal velocity than does the main species of ions.

Alternatively, the relative drift between two ion species might be driven by rf waves. $^{23}$ The drift could be accomplished by heating minority-species ions, traveling in one parallel direction, in the perpendicular velocity direction. That produces an ion drift much like heating tail electrons in the perpendicular direction produces a relative electron drift and the ECCD effect. In the case of minority ions, the heated ions collide less with the majority ions, so that, on average, the majority ions collide more with the unheated minority ions that travel in the opposite parallel direction. As a result, with momentum conserved, a relative drift is established between the two species of ions. This sets up the conditions for a current drive effect, known as minorityspecies current drive.

From a theoretical standpoint, minority-species current drive is somewhat less efficient than LHCD or ECCD. However, it does enjoy experimental verification on the JET tokamak, ${ }^{24}$ although with relatively low efficiency. It is thought nonetheless to be useful in controlling sawtooth instabilities. ${ }^{25,26}$

However, despite its poor efficiency, this current drive effect is important in that it employs ions rather than electrons. Thus, it can be used better in conjunction with means of establishing the so-called hot-ion mode. ${ }^{27}$ In the hot-ion mode, the ion temperatures are greater than the electron temperatures. A current drive method, such as minority-species current drive, that heats ions rather than electrons is more likely to maintain this temperature differential. The temperature differential might also be enabled by rf waves, as described in Sec. VI.

\section{ALPHA-PARTICLE ENVIRONMENT}

In a tokamak reactor, the current drive will have to be accomplished in the presence of alpha particles, the byproducts of the deuterium-tritium fusion reaction. The alpha-particle environment turns out to be qualitatively different from the environment that we encounter in present-day experiments, which is free of alpha particles. This is particularly true for lower hybrid wave propagation.

In fact, it was predicted that alpha particles might damp the lower hybrid wave, interfering with the LHCD effect in working reactors. ${ }^{28}$ This prediction was verified, with suggestions for mitigating the effect based upon radial diffusion of electrons. ${ }^{29}$ However, it remained unclear, in an environment of alpha-particle heating, whether the LHCD effect could work near the plasma center or whether the current near the plasma center would have to be driven by some other means. 
It turns out, however, that, since the alpha particles do tend to concentrate near the tokamak center, there may be free expansion energy that could be tapped. ${ }^{30}$ Then, the alpha particles need not damp the wave and may even amplify it. The wave amplification by alpha particles can be accompanied by wave damping by select electrons, leading to very efficient current drive. Moreover, if the alpha-particle energy were used to amplify a wave, and that wave were to damp on ions, the hot-ion mode might be enabled. This redirection of the alpha-particle power is known as alpha channeling. There is considerable utility in a tokamak reactor operating in the hot-ion mode. ${ }^{31}$

A significant amount of the alpha-particle power can, in fact, be redirected or channeled, by means of rf waves, either to ion heating or to current drive. This alphachanneling effect relies upon the coupling of diffusion in space to diffusion in energy. ${ }^{30}$ In the case of one wave, a strict constraint on diffusion can be arranged, so that it occurs only along a path that connects the dense energetic population of alpha particles in the tokamak center to the rarefied, cold population near the periphery. There are also advantages in using several waves at once. ${ }^{32}$

The mode-converted ion Bernstein wave has certain particularly attractive features for accomplishing the alpha-channeling effect, including a change in the sign of the parallel phase velocity after the mode conversion region is crossed. ${ }^{33}$ It can be used, therefore, to tap and channel much of the alpha-particle power. ${ }^{34}$ Since this power ends up being dissipated primarily in ions, it can enable not only the hot-ion mode but also current drive by ions. Even though the current drive effect by minority ions is not so efficient, the efficiency may not be important if the power is largely coming from the alpha particles. In addition, driving the current by heating the ions is consistent with achieving the hot-ion mode.

The alpha-channeling effect is highly speculative. In principle, if the diffusion paths work out as predicted, attractive reactor scenarios can be envisioned. ${ }^{35}$ Although there is no experimental evidence for the cooling effect, there is some for other aspects of the channeling effect, such as the diffusion paths. ${ }^{36}$

\section{ACKNOWLEDGMENTS}

This work was supported by the U.S. Department of Energy under contract DE-AC02-09CH11466. The author acknowledges the hospitality of the Weizmann Institute of Science, where he held a Weston Visiting Professorship for much of the time during which this manuscript was prepared.

\section{REFERENCES}

1. N. J. FISCH, "Theory of Current Drive in Plasmas," Rev. Mod. Phys., 59, l, 175 (1987); http://dx.doi.org/10.1103 /RevModPhys.59.175.
2. D. W. FAULCONER, "Current Drive," Fusion Sci. Technol., 53, 2T, 210 (2008).

3. E. WESTERHOF, "Non-Inductive Current Drive," Fusion Sci. Technol., 61, 2T, 312 (2012).

4. N. J. FISCH, "Confining a Tokamak Plasma with RfDriven Currents," Phys. Rev. Lett., 41, 13, 873 (1978); http:// dx.doi.org/10.1103/PhysRevLett.41.873.

5. T. H. STIX, Waves in Plasmas, 2nd ed., Springer-Verlag, New York (1992).

6. M. BRAMBILLA, Kinetic Theory of Plasma Waves: Homogeneous Plasmas, Oxford University Press, New York (1998).

7. J. M. RAX, Physique des plasmas, Dunod, Paris (2005).

8. J. M. RAX, "Physics of Landau Resonances, Cyclotron Resonances and Current Generation," Fusion Sci. Technol., 65, 10 (2014); http://dx.doi.org/10.13182/FST13-634.

9. C. F. F. KARNEY and N. J. FISCH, "Numerical Studies of Current Generation by Radio-Frequency Traveling Waves," Phys. Fluids, 22, 1817 (1979); http://dx.doi.org/10.1063 /1.862787.

10. N. J. FISCH and A. H. BOOZER, "Creating an Anisotropic Plasma Resistivity with Waves," Phys. Rev. Lett., 45, 720 (1980); http://dx.doi.org/10.1103/PhysRevLett.45.720.

11. D. J. H. WORT, “The Peristaltic Tokamak," Plasma Phys., 13, 258 (1971); http://dx.doi.org/10.1088/0032-1028/13/3/008.

12. N. J. FISCH and C. F. F. KARNEY, "Current Generation with Low-Frequency Waves," Phys. Fluids, 24, 27 (1981); http://dx.doi.org/10.1063/1.863243.

13. N. J. FISCH, "Current Generation in a Relativistic Plasma," Phys. Rev. A, 24, 3245 (1981); http://dx.doi.org /10.1103/PhysRevA.24.3245.

14. C. F. F. KARNEY and N. J. FISCH, "Currents Driven by Electron Cyclotron Waves," Nucl. Fusion, 21, 1549 (1981); http://dx.doi.org/10.1088/0029-5515/21/12/004.

15. C. F. F. KARNEY and N. J. FISCH, "Efficiency of Current Drive by Fast Waves," Phys. Fluids, 28, 116 (1985); http:// dx.doi.org/10.1063/1.865191.

16. C. F. F. KARNEY and N. J. FISCH, "Current in Wave Driven Plasmas," Phys. Fluids, 29, 180 (1986); http://dx.doi.org /10.1063/1.865975.

17. W. H. HOOKE, "Review of Experiments on Current Drive in Tokamaks by Means of RF Waves," Plasma Phys. Controlled Fusion, 26, 133 (1984); http://dx.doi.org/10.1088 /0741-3335/26/1A/312.

18. N. J. FISCH, “Conductivity of RF-Heated Plasma," Phys. Fluids, 28, 245 (1985); http://dx.doi.org/10.1063/1.865186.

19. N. J. FISCH and C. F. F. KARNEY, "Conversion of Wave Energy to Magnetic Field Energy in a Plasma Torus," Phys. Rev. Lett., 54, 897 (1985); http://dx.doi.org/10.1103 /PhysRevLett.54.897.

20. C. F. F. KARNEY, F. C. JOBES, and N. J. FISCH, "Comparison of the Theory and the Practice of Lower Hybrid 
Current Drive," Phys. Rev. A, 32, 2554 (1985); http://dx.doi.org /10.1103/PhysRevA.32.2554.

21. R. PRATER, "Heating and Current Drive by Electron Cyclotron Waves," Phys. Plasmas, 11, 2349 (2004); http://dx.doi.org/10.1063/1.1690762.

22. T. OHKAWA, "New Methods of Driving Plasma Current in Fusion Devices," Nucl. Fusion, 10, 185 (1970); http:// dx.doi.org/10.1088/0029-5515/10/2/012.

23. N. J. FISCH, "Current Generation by Minority-Species Heating," Nucl. Fusion, 21, 15 (1981); http://dx.doi.org /10.1088/0029-5515/21/1/002.

24. M. J. MANTSINEN et al, "Application of ICRF Waves in Tokamaks Beyond Heating," Plasma Phys. Controlled Fusion, 45, A445 (2003); http://dx.doi.org/10.1088/0741-3335/45/12A $/ 028$.

25. M. LAXABACK and T. HELLSTEN, "Modelling of Minority Ion Cyclotron Current Drive During the Activated Phase of ITER," Nucl. Fusion, 45, 1510 (2005); http:// dx.doi.org/10.1088/0029-5515/45/12/006.

26. L. G. ERIKSSON et al, "On Ion Cyclotron Current Drive for Sawtooth Control," Nucl. Fusion, 46, S951 (2006); http://dx.doi.org/10.1088/0029-5515/46/10/S12.

27. J. F. CLARKE, "Hot-Ion-Mode Ignition in a Tokamak Reactor," Nucl. Fusion, 20, 5, 563 (1980); http://dx.doi.org /10.1088/0029-5515/20/5/005.

28. K. L. WONG and M. ONO, "Effects of Ion-Cyclotron Harmonic Damping on Current Drive in the Lower Hybrid Frequency-Range," Nucl. Fusion, 24, 5, 615 (1984); http://dx.doi.org/10.1088/0029-5515/24/5/008.
29. N. J. FISCH and J. M. RAX, "Current Drive by Lower Hybrid Waves in the Presence of Energetic Alpha-Particles," Nucl. Fusion, 32, 4, 549 (1992); http://dx.doi.org/10.1088/00295515/32/4/I02.

30. N. J. FISCH and J. M. RAX, "Interaction of Energetic Alpha-Particles with Intense Lower Hybrid Waves," Phys. Rev. Lett., 69, 612 (1992); http://dx.doi.org/10.1103 /PhysRevLett.69.612.

31. N. J. FISCH and M. C. HERRMANN, "Utility of Extracting Power from Alpha Particles by Waves," Nucl. Fusion, 34, 1541 (1994); http://dx.doi.org/10.1088/0029-5515 /34/12/I01.

32. N. J. FISCH and M. C. HERRMANN, "Alpha Power Channelling with Two Waves," Nucl. Fusion, 35, 12, 1753 (1995); http://dx.doi.org/10.1088/0029-5515/35/12/I40.

33. E. J. VALEO and N. J. FISCH, "Excitation of Large- $k_{\theta}$ Ion-Bernstein Waves in Tokamaks," Phys. Rev. Lett., 73, 3536 (1994); http://dx.doi.org/10.1103/PhysRevLett.73.3536.

34. N. J. FISCH, "Alpha Power Channeling Using IonBernstein Waves," Phys. Plasmas, 2, 2375 (1995); http:// dx.doi.org/10.1063/1.871454.

35. M. C. HERRMANN and N. J. FISCH, "Cooling Energetic $\alpha$ Particles in a Tokamak with Waves," Phys. Rev. Lett., 79, 1495 (1997); http://dx.doi.org/10.1103/PhysRevLett.79.1495.

36. N. J. FISCH and M. C. HERRMANN, "A Tutorial on Alpha-Channelling," Plasma Phys. Controlled Fusion, 41, 3A, A221 (1999); http://dx.doi.org/10.1088/0741-3335/41/3A/015. 